\title{
SUPPLEMENTAL INFORMATION: \\ IN SILICO DISCOVERY OF MULTISTEP CHEMISTRY INITIATED BY A CONICAL INTERSECTION: THE CHALLENGING CASE OF DONOR ACCEPTOR STENHOUSE ADDUCTS
}

\author{
David M. Sanchez, ${ }^{1,2, \#}$ Umberto Raucci, ${ }^{1,2, \&}$ and Todd J. Martínez ${ }^{1,2}$ \\ ${ }^{1}$ Stanford PULSE Institute, SLAC National Accelerator Laboratory, Menlo Park, CA, 94025 \\ ${ }^{2}$ Department of Chemistry, Stanford University, Stanford, CA, 94305
}

Supplementary Information table of contents:

I. Computational Details

II. Population Dynamics from ab initio Multiple Spawning

III. Classifying Isomers from Structural Dynamics on $\mathrm{S}_{0}$

Figure S1. Molecular Orbitals of the Open-Form DASA

Figure S2. Binning Criteria for $\mathrm{S}_{0}$ Isomers

Figure S3. Representative Trajectory for A to B Conversion

Figure S4. Representative Trajectory for $\mathbf{B}$ to B" Conversion

Figure S5. Representative Trajectory for B" to B"' Conversion

Movie Descriptions. DASA Excited- and Ground-State Chemistry Movies 


\section{Computational Details}

The full photoinitiated ring-closing mechanism of Meldrum's acid $1^{\text {st }}$ generation DonorAcceptor Stenhouse Adducts (DASA) in the gas phase is investigated using ab initio multiple spawning $^{1-3}$ (AIMS) and Born-Oppenheimer molecular dynamics (BOMD). We simulate the first $10 \mathrm{ps}$ of ultrafast dynamics for Meldrum acid $1^{\text {st }}$ generation DASA by: 1) using AIMS to propagate the initial wavepacket for the first $5 \mathrm{ps}$ or until all population has returned to the ground state, 2) stopping TBFs on the ground state when they are decoupled from other TBFs (off-diagonal elements of the Hamiltonian become small), and 3) adiabatically continuing these stopped TBFs using the positions and momenta from the last frame in AIMS as initial conditions for discovery-based BOMD. Each of the $186 \mathrm{TBFs}$ were propagated up to $10 \mathrm{ps}$ for a total of $\sim 2 \mathrm{~ns}$, resulting in one of the largest discovery-based fully $a b$ initio nonadiabatic molecular dynamics studies to date. As a result of this 2ns AIMS/AIMD, we were able to identify several intermediates: a few have been experimentally observed (A', B', $\mathbf{B}^{\prime \prime \prime}$, and EZZZ), while we propose many for the first time. Furthermore, we show the natural transformation of these intermediates in real time, which until now, has yet to be shown for the main photoswitching pathway. Our discovered intermediates were deemed stable from a frequency analysis and their respective pathways from the AIMS/AIMD simulations agreed well with those computed by geodesic interpolation. ${ }^{4}$ The Cartesian coordinates of all optimized structures and the main photoswitching pathway from geodesic interpolation are provided in the SI-Files.

For the AIMS dynamics, we used GPU-accelerated State-Averaged Complete Active Space Self Consistent Field theory ${ }^{5-7}$ (SA-CASSCF) consisting of an active space of two electrons in two orbitals determined to minimize the average energy of the lowest three singlet states, in conjunction with the $6-31 \mathrm{G}^{* *}$ basis set, i.e. SA3-CAS(2,2)SCF/6-31G**. The first 
three singlet states $\left(\mathrm{S}_{0}, \mathrm{~S}_{1}\right.$, and $\left.\mathrm{S}_{2}\right)$ are included in the dynamics. The description of the species involved in the excited state chemistry (e.g., $S_{1}$ minima, $\alpha / \beta$ Minimum Energy Conical Intersection), were validated in our previous work by direct comparison to XMSPT2 (which includes dynamic electron correlation effects). ${ }^{8}$ We also note that the intersections obtained by $\mathrm{SA} 3-\mathrm{CAS}(2,2) \mathrm{SCF} / 6-31 \mathrm{G}^{* *}$ for this molecule are in good agreement with larger active space CASSCF and CASPT2 results reported by García-Iriepa and coworkers. ${ }^{9}$

The adaptive timestep was set to $0.48 \mathrm{fs}(20 \mathrm{au})$ (reduced to $0.12 \mathrm{fs}$ ( $5 \mathrm{au}$ ) in regions with large nonadiabatic coupling) and used to propagate the centers of the trajectory basis functions (TBFs). A coupling threshold of 0.01 au (scalar product of nonadiabatic coupling and velocity vectors) initiates spawning events generating new TBFs on different electronic states. Population transfer between TBFs is described by solving the time-dependent Schrödinger equation in the time-evolving TBF basis set. We refer the reader elsewhere for details regarding these AIMS simulations. ${ }^{8}$

For the discovery-based BOMD dynamics on the ground state, we employ unrestricted Density Functional Theory (DFT) with the Perdew-Burke-Ernzerhof hybrid exchange-correlation functional, ${ }^{10}$ i.e uPBE0-D3/6-31G**. The BOMD was sampled according to the microcanonical ensemble (NVE) (i.e. fixed number of atoms, volume, and energy) using a fixed timestep of 0.5 fs. All electronic structure calculations (i.e. energies, gradients, and nonadiabatic coupling vectors $(\mathrm{NACV})$ ) are performed with the TeraChem electronic structure package. ${ }^{11-13} \mathrm{~A}$ total of $236 \mathrm{TBF}$ are propagated during the course of the entire simulation, with 186 of these being adiabatically continued on the ground state with DFT. Cartesian coordinates of all $\mathrm{S}_{0}$ intermediates discovered during the adiabatic dynamics were optimized at the uPBE0-D3/6$31 \mathrm{G}^{* *}$ level of theory and included in the SI-Files.

Sanchez, D. M., Raucci, U. et al. - DASA Ground-State Dynamics - Page 3 
The uPBE method provides a more accurate description for the ground electronic state than CASSCF (specifically for the barriers between different intermediates). Thus, our scheme matches an accurate description of the excited state and initial ground state dynamics (which uPBE cannot provide since it cannot describe conical intersections) to an accurate description of the longer time dynamics on the ground state. This method (treating short-time excited state dynamics with CASSCF and longer time ground state dynamics with DFT) has been successfully applied previously. ${ }^{14-16}$

\section{Ab Initio Multiple Spawning}

Ab initio multiple spawning (AIMS) is a nonadiabatic dynamics algorithm aimed at describing photodynamical processes involving multiple electronic states using a time-dependent basis set. In the following, we present a brief introduction to the working equations of AIMS and refer the reader elsewhere for a more a more detailed discussion. ${ }^{17}$ The exact molecular wavefunction can be separated into electronic and nuclear contributions using the Born-Huang representation:

$$
\Psi(\mathbf{r}, \mathbf{R}, t)=\sum_{I} \chi_{I}(\mathbf{R}, t) \phi_{I}(\mathbf{r} ; \mathbf{R})
$$

where $\chi_{I}(\mathbf{R}, t)$ denotes the time-dependent nuclear wavefunction for electronic state I and $\phi_{I}(\mathbf{r} ; \mathbf{R})$ is the electronic wavefunction for state I at nuclear configuration $\mathbf{R}$. In the adiabatic representation, $\phi_{I}(\mathbf{r} ; \mathbf{R})$ is expanded into an orthonormal electronic basis consisting of eigenfunctions of the time-independent electronic Schrödinger equation (TIESE) parametrically dependent on nuclear configuration $\mathbf{R}$. Under the AIMS ansatz, $\chi_{I}(\mathbf{R}, t)$ is represented as a superposition of frozen Gaussian functions called trajectory basis functions (TBF): 


$$
\chi_{I}(\mathbf{R}, t)=\sum_{k=1}^{N_{t}(t)} c_{k}^{I}(t) \chi_{k}^{I}\left(\mathbf{R} ; \overline{\mathbf{R}}_{k}^{I}(t), \overline{\mathbf{P}}_{k}^{I}(t), \bar{\gamma}_{k}^{I}(t), \alpha_{k}^{I}\right)
$$

where $N_{I}(t)$ is the total number of TBFs on electronic state I, $c_{k}^{I}(t)$ is the time-dependent complex coefficient of the $k$ th TBF on electronic state I, $\boldsymbol{\alpha}_{k}^{I}$ is the frozen TBF width, and $\chi_{k}^{I}(\ldots)$ is a multidimensional frozen Gaussian that is expressed as a product of one-dimensional Gaussian functions corresponding to the $3 \mathrm{~N}$ nuclear degrees of freedom. In AIMS, each TBF evolves adiabatically along one Born-Oppenheimer electronic surface. The time-dependent positions and momenta, $\left(\bar{R}_{\rho_{k}}^{I}(t), \bar{P}_{\rho_{k}}^{I}(t)\right)$, of the TBFs are propagated classically according to Hamilton's equations of motion on the given electronic state:

$$
\begin{gathered}
\frac{\partial \overline{\mathrm{R}}_{\rho_{k}}^{I}(t)}{\partial t}=\frac{\overline{\mathrm{P}}_{\rho_{k}}^{I}(t)}{m_{\rho}} \\
\frac{\partial \bar{P}_{\rho_{k}}^{I}(t)}{\partial t}=-\left.\frac{\partial E_{I}(\mathbf{R})}{\partial R_{\rho_{k}}}\right|_{\bar{R}_{\rho_{k}}^{I}(t)}
\end{gathered}
$$

where $m_{\rho}$ is the mass for the $\rho$ th nuclear coordinate and $E_{I}(\mathbf{R})$ is the electronic energy of state I with nuclear configuration $\mathbf{R}$. The nuclear phase, $\bar{\gamma}_{k}^{I}(t)$, is propagated semiclassically according to the classical Lagrangian:

$$
\frac{\partial \bar{\gamma}_{k}^{I}}{\partial t}=\sum_{\rho=1}^{3 N} \frac{\left(\bar{P}_{\rho_{k}}^{I}(t)\right)^{2}}{2 m_{\rho}}-E_{I}\left(\overline{\mathbf{R}}_{k}^{I}(t)\right)
$$

The evolution of the time-dependent amplitudes is governed by the time-dependent Schrodinger equation (TDSE) written in matrix form as: 


$$
\frac{d \mathbf{C}^{I}(t)}{d t}=-i\left(\mathbf{S}_{I I}^{-1}\right)\left\{\left[\mathbf{H}_{I I}-i \dot{\mathbf{S}}_{I I}\right] \mathbf{C}^{I}+\sum_{J \neq I} \mathbf{H}_{I J} \mathbf{C}^{J}\right\}
$$

where $\mathbf{S}$ and $\dot{\mathbf{S}}$ are the nuclear overlap matrix and its right-acting time-derivative, respectively, and $\mathbf{H}$ is the Hamiltonian. The matrix elements of the Hamiltonian are computed using the zeroth-order saddle-point (SP) approximation, which involves evaluating the zeroth-order Taylor expansion of the PES and/or nonadiabatic coupling vector (NACV) around the centroid position between pairs of TBFs. In addition to the SP approximation, the independent first generation (IFG) approximation is used to describe the initial nuclear wavepacket at time $\mathrm{t}=0$ as a swarm of independent initial TBFs each with their own positions and momenta sampled from a harmonic Wigner distribution, i.e. initial conditions (IC). Unlike the initial TBFs, the spawned TBFs from each IC remain coupled during the course of the dynamics and their separation naturally accounts for decoherence of the nuclear wavefunction on multiple electronic states.

\section{Classifying Isomers from Structural Dynamics on $\mathbf{S}_{0}$}

The natural evolution of the wavepacket dynamics can be followed by binning geometries along ground-state TBFs into one of the DASA photoproducts $\left(\mathbf{A}, \mathbf{A}^{\prime}, \mathbf{A}^{\prime \prime}, \mathbf{B} / \mathbf{B}^{\prime}, \mathbf{B}^{\prime \prime}, \mathbf{B}^{\prime \prime \prime}\right.$, EEZE, EZEE, EZZE, EEEE, EZZZ, and ZEZZ), following previous studies. ${ }^{14}$ Snapshots taken every 50fs along all 186-ground state DFT TBFs were binned into one of the isomers based on dihedral angles $(\alpha, \beta, \gamma, \delta, \omega)$ and atomic distances $\left(\mathrm{C}_{2}-\mathrm{C}_{6}\right.$ and $\left.\mathrm{N}-\mathrm{H}_{\mathrm{a}}\right)$. For details on the binning criteria, see Supplementary Fig. S2. Due to the ground state TBFs being sufficiently uncoupled from all others, the total population of a specific isomer $L$ at time $t$ on the groundstate, $P_{L}(t)$ is computed by: 


$$
P_{L}(t)=\frac{1}{N_{I C}} \sum_{M=1}^{N_{C L}}\left[\frac{\sum_{k}^{N_{T B F}^{M}(t)} c_{k, M}^{*}(t) c_{k, M}(t) \delta\left(L, I\left(\bar{R}_{k, M}(t)\right)\right)}{\sum_{k}^{N_{I B F}^{M}(t)} c_{k, M}^{*}(t) c_{k, M}(t)}\right]
$$

where $L$ is defined as one of (A, $\mathbf{A}^{\prime}, \mathbf{A}^{\prime \prime}, \mathbf{B} / \mathbf{B}^{\prime}, \mathbf{B}^{\prime \prime}, \mathbf{B} "$ ", EEZE, EZEE, EZZE, EEEE, EZZZ, and ZEZZ), $c_{k, M}(t)$ is the amplitude of the $k$ th TBF and $M$ th IC, $N_{I C}$ is the total number of initial conditions, and $\delta(\ldots)$ is a Kronecker-delta function, and $I(R)$ represents the isomer classification of the geometry given by $R$. 

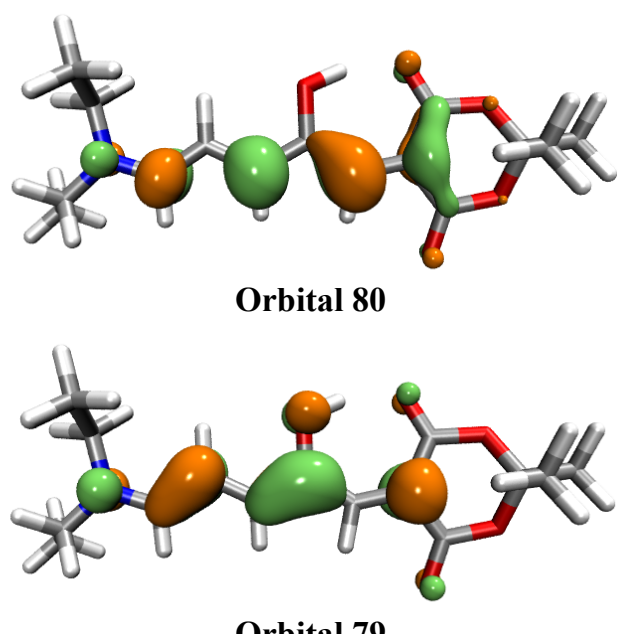

Orbital 79

Figure S1. The SA3-CAS $(2,2) \mathrm{SCF} / 6-31 \mathrm{G}^{* *}$ natural orbitals at the $\mathrm{S}_{0}$ minimum. Orange and green correspond to 0.05 and- $0.05 \mathrm{e}-/ \AA^{3}$ isovalues, respectively. 


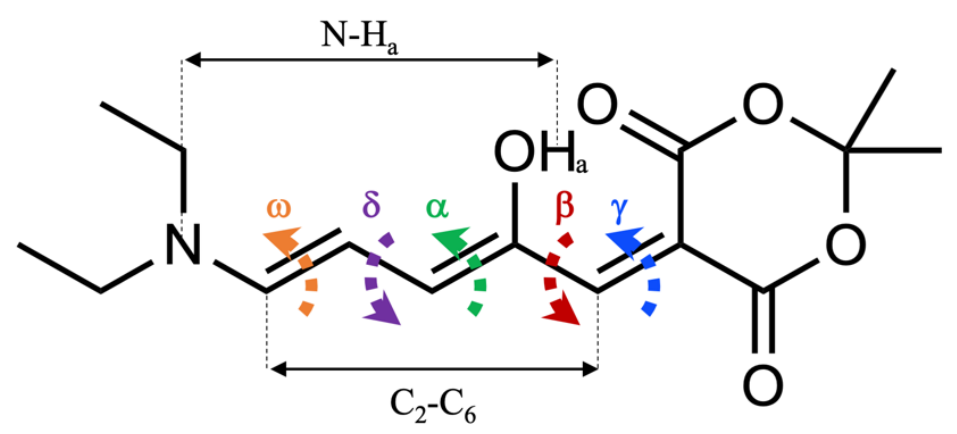

\begin{tabular}{|c|c|c|c|c|c|c|c|}
\hline \multirow{2}{*}{ Isomer } & \multicolumn{7}{|c|}{ Binning Criteria } \\
\cline { 2 - 8 } & $\begin{array}{c}\alpha \\
\left({ }^{\circ}\right)\end{array}$ & $\begin{array}{c}\beta \\
\left({ }^{\circ}\right)\end{array}$ & $\begin{array}{c}\gamma \\
\left({ }^{\circ}\right)\end{array}$ & $\begin{array}{c}\delta \\
\left({ }^{\circ}\right)\end{array}$ & $\begin{array}{c}\omega \\
\left({ }^{\circ}\right.\end{array}$ & $\begin{array}{c}\mathrm{C}_{2}-\mathrm{C}_{6} \\
(\AA)\end{array}$ & $\begin{array}{c}\mathrm{N}^{\circ}-\mathrm{H}_{\mathrm{a}} \\
(\AA)\end{array}$ \\
\hline $\mathbf{A}$ & $\geq 100$ & $\geq 100$ & $\geq 100$ & $\geq 100$ & $\geq 100$ & $\geq 2.0$ & $\geq 2.0$ \\
\hline $\mathbf{A}^{\prime}$ & $\leq \mathbf{8 0}$ & $\geq 100$ & $\geq 100$ & $\geq 100$ & $\geq 100$ & $\geq 2.0$ & $\geq 2.0$ \\
\hline $\mathbf{A}^{\prime \prime}$ & $\leq \mathbf{8 0}$ & $\geq 100$ & $\geq 100$ & $\leq \mathbf{8 0}$ & $\geq 100$ & $\geq 2.0$ & $\geq 2.0$ \\
\hline $\mathbf{B} / \mathbf{B}^{\prime}$ & $\leq \mathbf{8 0}$ & $\geq 100$ & $\geq 100$ & $\leq \mathbf{8 0}$ & $\geq 100$ & $<\mathbf{2 . 0}$ & $\geq 2.0$ \\
\hline $\mathbf{B}^{\prime \prime}$ & $\leq \mathbf{8 0}$ & $\geq 100$ & $\leq \mathbf{8 0}$ & $\leq \mathbf{8 0}$ & $\geq 100$ & $<\mathbf{2 . 0}$ & $\geq 2.0$ \\
\hline $\mathbf{B}^{\prime \prime \prime}$ & $\leq \mathbf{8 0}$ & $\geq 100$ & $\leq \mathbf{8 0}$ & $\leq \mathbf{8 0}$ & $\geq 100$ & $<\mathbf{2 . 0}$ & $<\mathbf{2 . 0}$ \\
\hline EEZE & $\geq 100$ & $\leq \mathbf{8 0}$ & $\geq 100$ & $\geq 100$ & $\geq 100$ & $\geq 2.0$ & $\geq 2.0$ \\
\hline EEEE & $\leq \mathbf{8 0}$ & $\leq \mathbf{8 0}$ & $\geq 100$ & $\geq 100$ & $\geq 100$ & $\geq 2.0$ & $\geq 2.0$ \\
\hline EZZZ & $\geq 100$ & $\geq 100$ & $\geq 100$ & $\leq \mathbf{8 0}$ & $\geq 100$ & $\geq 2.0$ & $\geq 2.0$ \\
\hline ZEZZ & $\geq 100$ & $\geq 100$ & $\geq 100$ & $\geq 100$ & $\leq 80$ & $\geq 2.0$ & $\geq 2.0$ \\
\hline EZZE & $\leq \mathbf{8 0}$ & $\leq \mathbf{8 0}$ & $\geq 100$ & $\geq 100$ & $\geq 100$ & $\geq 2.0$ & $\geq 2.0$ \\
\hline EZEE & $\leq \mathbf{8 0}$ & $\leq \mathbf{8 0}$ & $\geq 100$ & $\leq \mathbf{8 0}$ & $\geq 100$ & $\geq 2.0$ & $\geq 2.0$ \\
\hline
\end{tabular}

Figure S2. Dihedral angle and atomic distance labels for structural parameters in DASA. Binning criteria used to classify isomers discovered during the ground-state adiabatic dynamics. 


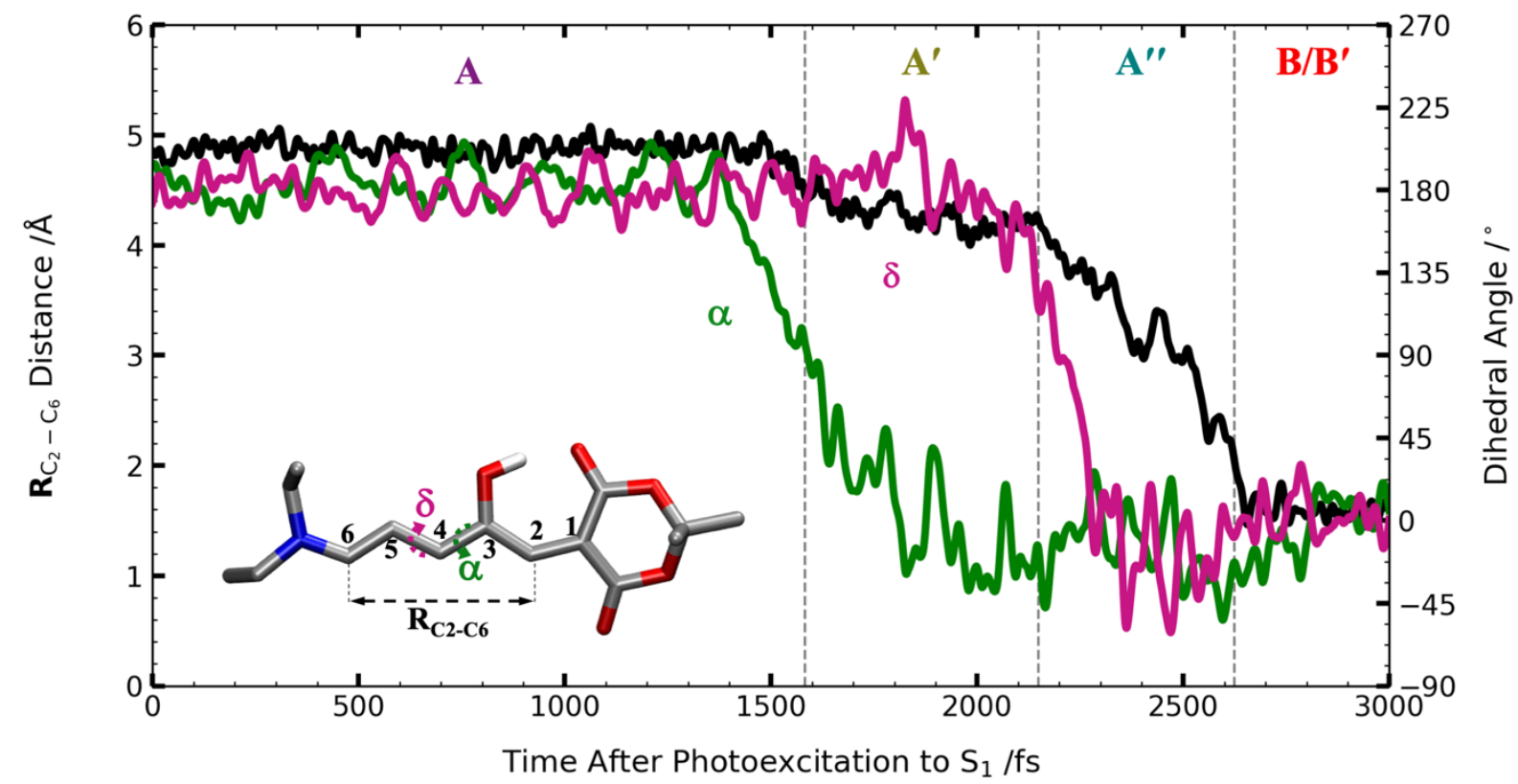

Figure S3. The complete $4 \pi$-electrocyclization pathway (characterized by the time evolution of $\alpha, \delta$, and the $\mathrm{C}_{2}-\mathrm{C}_{6}$ distance) for a representative trajectory of the AIMS/BOMD simulation. A three-step mechanism can be clearly identified: 1) isomerization around $\alpha$ forms $\mathbf{A}^{\prime} 2$ ) subsequent rotation of $\delta$ forms $\mathbf{A}^{\prime \prime} 3$ ) shortening of $\mathrm{C}_{2}-\mathrm{C}_{6}$ distance leads to $\mathbf{B}$. In the first 1.6ps, the molecule exists in the $\mathbf{A}$ configuration, where both $\alpha$ and $\delta$ are approximately $180^{\circ}$ and the $\mathrm{C}_{2}-\mathrm{C}_{6}$ distance oscillates around $4.8 \AA$. Upon relaxation through the $\mathrm{S}_{1} / \mathrm{S}_{0} \mathrm{CI}$ at $\sim 1.6 \mathrm{ps}, \alpha$ begins to twist $90^{\circ}$ resulting in a shortening of the $\mathrm{C}_{2}-\mathrm{C}_{6}$ distance by $0.5 \AA$ and the formation of $\mathbf{A}^{\prime}$. At approximately $2.2 \mathrm{ps}$, the $\delta$ angle begins to twist leading to the formation of $\mathbf{A}^{\prime \prime}$. Once $\mathbf{A}^{\prime \prime}$ is formed at $\sim 2.6 \mathrm{ps}$, the $\mathrm{C}_{2}-\mathrm{C}_{6}$ distance shortens to $\sim 1.55 \AA$, providing an optimal arrangement for the carbon-carbon bond formation leading to the closed cyclopentanone form $\mathbf{B}$. 
a)

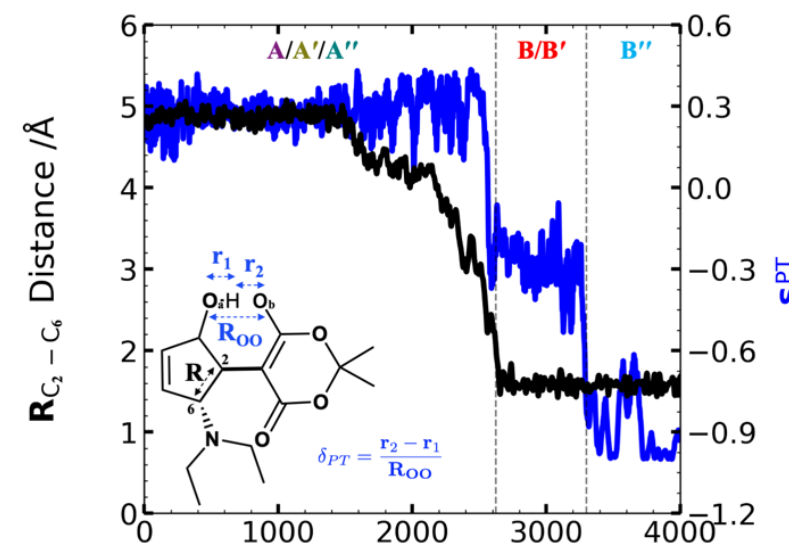

Time After Photoexcitation to $\mathrm{S}_{1} / \mathrm{fs}$ b)

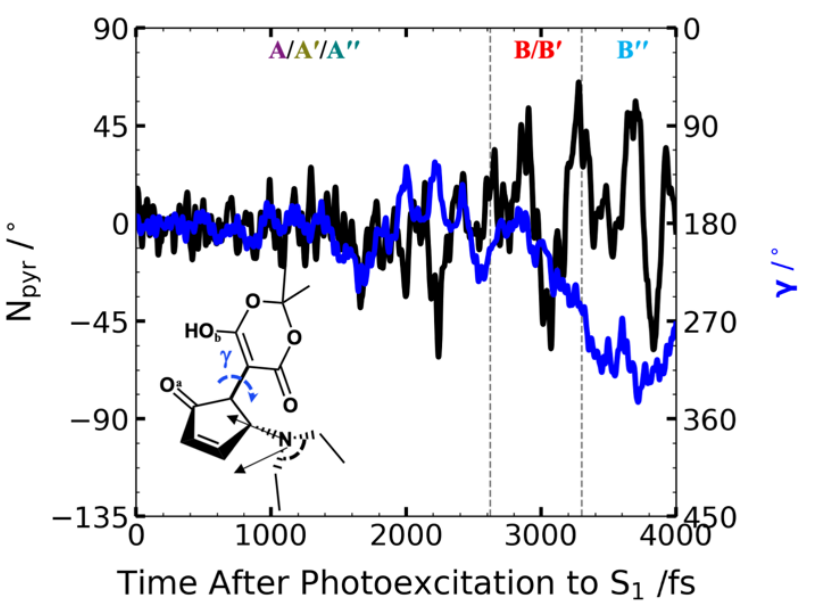

Figure S4. In a, the time evolution of the $\mathrm{C}_{2}-\mathrm{C}_{6}$ distance and proton transfer (PT) coordinate between the hydroxyl and neighboring carbonyl oxygens $\left(\delta_{O H O}^{P T}\right)$ shows a highly coupled motion, resulting in a clear distinction between $\mathbf{A} / \mathbf{A}^{\prime} / \mathbf{A}^{\prime \prime}$ and B-type intermediates. In the first $2.5 \mathrm{ps}$, $\delta_{O H O}^{P T}$ oscillates around 0.3 (with small fluctuations of the hydrogen bond), and a stable $\mathrm{O}_{\mathrm{a}} \mathrm{H}$ bond is observed in the $\mathbf{A} / \mathbf{A}^{\prime} / \mathbf{A}^{\prime \prime}$ species. The formation of $\mathbf{A}^{\prime \prime}$ leads to a significant decrease of the $\mathrm{C}_{2}$ $\mathrm{C}_{6}$ distance, which is accompanied by the PT from the hydroxyl to the carbonyl group at $2.6 \mathrm{ps}$ (i.e. $\delta_{O H O}^{P T}$ changes sign). Next, the decrease in $\delta_{O H O}^{P T}$ marks the formation of $\mathbf{B}$ when the hydrogen bound to $\mathrm{O}_{\mathrm{a}}$ transfers to $\mathrm{O}_{\mathrm{b}}$. Around $3.3 \mathrm{ps}$, a second jump in $\delta_{O H O}^{P T}$ is observed from -0.3 to -0.9 when the $\mathrm{O}_{\mathrm{a}}-\mathrm{O}_{\mathrm{b}}$ hydrogen bond is disrupted due to rotation in $\gamma$, leading to the formation of $\mathbf{B}$ ". In $\mathbf{b}$, we show the time evolution of $\mathrm{N}_{\mathrm{pyr}}$ and its coupling with the $\gamma$ rotation which reveals that the nitrogen freely inverts for all $\mathbf{A}$ and $\mathbf{B}$ intermediates due to excess energy via dynamics through a CI. Large fluctuations of the $\mathrm{N}_{\text {pyr }}$ angle $\left( \pm 60^{\circ}\right)$ are observed in $\mathbf{B}$, making it difficult to distinguish $\mathrm{B}$ conformers based on $\mathrm{N}_{\text {pyr }}$ alone. This refines the mechanism hypothesized by Zulfikri et al. where a $\mathbf{B}^{\prime}$ intermediate (characterized by inversion about $\mathrm{N}$ ) has been proposed. ${ }^{18}$ 


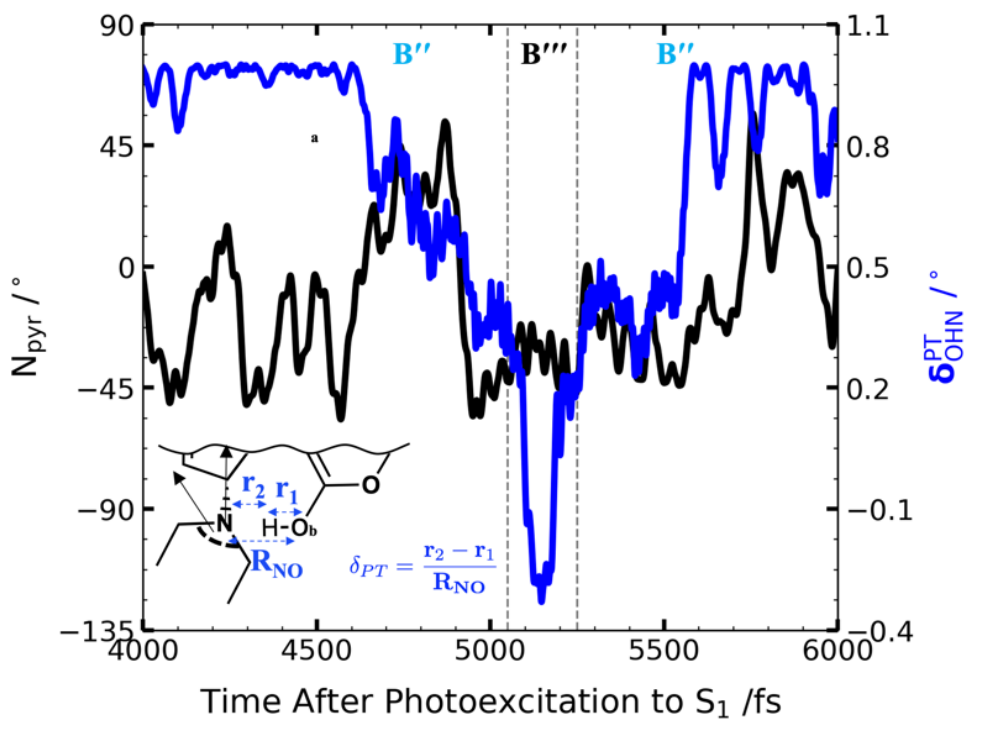

Figure S5. The coupling between $\mathrm{N}_{\mathrm{pyr}}$ and the PT coordinate between $\mathrm{O}_{\mathrm{b}}$ and the nitrogen $\left(\delta_{O H N}^{P T}\right)$ is evident in in the above figure. When the nitrogen is pyramidalized away from acceptor (positive $\left.\mathrm{N}_{\mathrm{pyr}}\right)$ poor interaction with the hydrogen inhibits the PT reaction $\left(\delta_{O H N}^{P T}\right.$ oscillates around 0.5 ). At $\sim 4.6 \mathrm{ps}$ after the photoexcitation, the nitrogen inverts (negative $\mathrm{N}_{\mathrm{pyr}}$ ) and $\delta_{O H N}^{P T}$ decreases to 0.35 . This orientation strengthens the hydrogen bond between the nitrogen and the hydrogen of $\mathrm{O}_{\mathrm{b}}$ via more favorable orbital overlap of the nitrogen lone pair and hydrogen, leading to the subsequent formation of $\mathbf{B}^{\prime \prime}$. However, the back-proton transfer from the nitrogen to $\mathrm{O}_{\mathrm{b}}$ is also observed after $5.25 \mathrm{ps}$, leading to a fast interconversion between $\mathbf{B}^{\prime \prime}$ and $\mathbf{B} "$ '. 


\section{Movie Descriptions: DASA Excited- and Ground-State Chemistry Movies}

(Movie: A-B'"-Full-Pathway.mov) This movie was generated from the coordinate expectation value of a single AIMS trajectory basis function propagating first on $\mathrm{S}_{1}$ and then

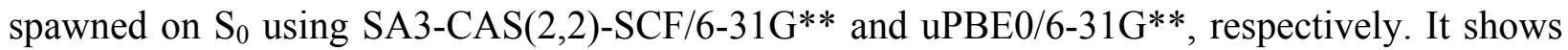
the full DASA $1^{\text {st }}$ generation photoswitching mechanism in isolation discovered using free NVE dynamics with no enhanced sampling techniques. DASA starts in the open linear from, $\mathbf{A}$, at equilibrium on the ground-state. Upon excitation with light, DASA is promoted to an electronically excited-state, where it undergoes nonradiative relaxation via $\alpha$ or $\beta$ nonradiative relaxations channels. On the ground-state, several pathways are accessible due to the absorbed energy of the photon. After forming $\mathbf{A}$, a second isomerization takes place around $\delta$ resulting in the formation of $\mathbf{A}^{\prime \prime}$. DASA then undergoes a concerted proton transfer and C-C bond formation, forming B. Lastly, DASA eventually twists around $\gamma$ to form B", which correctly orients the proton with the donor group nitrogen. The movie end with the transfer of the proton to the nitrogen forming the zwitterionic DASA intermediate, B'". All subsequent steps shown in Figure 2 were generated in the same way and are labeled accordingly (i.e. reactantproduct.mov). 


\section{References}

(1) Ben-Nun, M.; Martínez, T. J. Nonadiabatic molecular dynamics: Validation of the multiple spawning method for a multidimensional problem. J. Chem. Phys. 1998, 108, 7244.

(2) Ben-Nun, M.; Quenneville, J.; Martínez, T. J. Ab Initio Multiple Spawning: Photochemistry from First Principles Quantum Molecular Dynamics. J. Phys. Chem. A 2000, 104, 5161.

(3) Ben-Nun, M.; Martínez, T. J. Ab initio quantum molecular dynamics. Adv. Chem. Phys. 2002, 121, 439.

(4) Zhu, X.; Thompson, K. C.; Martínez, T. J. Geodesic interpolation for reaction pathways. $J$. Chem. Phys. 2019, 150, 164103.

(5) Snyder, J. W., Jr.; Fales, B. S.; Hohenstein, E. G.; Levine, B. G.; Martinez, T. J. A directcompatible formulation of the coupled perturbed complete active space self-consistent field equations on graphical processing units. J. Chem. Phys. 2017, 146, 174113.

(6) Snyder, J. W.; Curchod, B. F. E.; Martínez, T. J. GPU-Accelerated State-Averaged Complete Active Space Self-Consistent Field Interfaced with Ab Initio Multiple Spawning Unravels the Photodynamics of Provitamin D3. J. Phys. Chem. Lett. 2016, 7, 2444.

(7) Hohenstein, E. G.; Luehr, N.; Ufimtsev, I. S.; Martinez, T. J. An atomic orbital-based formulation of the complete active space self-consistent field method on graphical processing units. J. Chem. Phys. 2015, 142, 224103.

(8) Sanchez, D. M.; Raucci, U.; Ferreras, K. N.; Martínez, T. J. Putting Photomechanical Switches to Work: An Ab Initio Multiple Spawning Study of Donor-Acceptor Stenhouse Adducts. J. Phys. Chem. Lett. 2020, 11, 7901.

(9) García-Iriepa, C.; Marazzi, M.; Sampedro, D. From Light Absorption to Cyclization: Structure and Solvent Effects in Donor-Acceptor Stenhouse Adducts. ChemPhotoChem 2019, 3, 866.

(10) Adamo, C.; Barone, V. Toward reliable density functional methods without adjustable parameters: The PBE0 model. J. Chem. Phys. 1999, 110, 6158.

(11) Ufimtsev, I. S.; Martinez, T. J. Quantum Chemistry on Graphical Processing Units. 2. Direct Self-Consistent-Field Implementation. J. Chem. Theory Comput. 2009, 5, 1004.

(12) Ufimtsev, I. S.; Martinez, T. J. Quantum chemistry on graphical processing units. 3. Analytical energy gradients, geometry optimization, and first principles molecular dynamics. $J$. Chem. Theory Comput. 2009, 5, 2619.

(13) Ufimtsev, I. S.; Martínez, T. J. Quantum Chemistry on Graphical Processing Units. 1. Strategies for Two-Electron Integral Evaluation. J. Chem. Theory Comput. 2008, 4, 222.

(14) Wolf, T. J. A.; Sanchez, D. M.; Yang, J.; Parrish, R. M.; Nunes, J. P. F.; Centurion, M.; Coffee, R.; Cryan, J. P.; Gühr, M.; Hegazy, K.; Kirrander, A.; Li, R. K.; Ruddock, J.; Shen, X.; Vecchione, T.; Weathersby, S. P.; Weber, P. M.; Wilkin, K.; Yong, H.; Zheng, Q.; Wang, X. J.; Minitti, M. P.; Martínez, T. J. The photochemical ring-opening of 1,3-cyclohexadiene imaged by ultrafast electron diffraction. Nature Chem. 2019, 11, 504.

(15) Snyder Jr, J. W.; Curchod, B. F.; Martínez, T. J. GPU-accelerated state-averaged complete active space self-consistent field interfaced with ab initio multiple spawning unravels the photodynamics of provitamin D3. J. Phys. Chem. Lett. 2016, 7, 2444.

(16) Mignolet, B.; Curchod, B. F.; Martínez, T. J. Rich Athermal Ground-State Chemistry Triggered by Dynamics through a Conical Intersection. Ang. Chem. Int. Ed. 2016, 55, 14993.

(17) Curchod, B. F. E.; Martínez, T. J. Ab Initio Nonadiabatic Quantum Molecular Dynamics. Chem. Rev. 2018, 118, 3305. 
(18) Zulfikri, H.; Koenis, M. A. J.; Lerch, M. M.; Di Donato, M.; Szymański, W.; Filippi, C.; Feringa, B. L.; Buma, W. J. Taming the Complexity of Donor-Acceptor Stenhouse Adducts: Infrared Motion Pictures of the Complete Switching Pathway. J. Amer. Chem. Soc. 2019, 141, 7376. 